\title{
Évangélisation et Mission: enjeux et défis en perspective libanaise
}

\author{
Evangelização e missão: questões e desafios em \\ perspectiva libanesa
}

Ziad Fahed

\begin{abstract}
This paper explores how the Christian community in Lebanon perceives the mission and evangelization within the Middle Eastern context. Despite the tensions and the challenges related to the repeated wars and violent extremism that are surrounding the church in Lebanon, the Christian community is called to renew the process of deep understanding of its own mission. The teachings revealed through Evangelii Nunitiandi (Paul VI) till Evangelii Gaudium (Pope Francis) enrich the local Christian experience and redirect its mission to be perceived in its glocal context, here and now! This mission is called to surpass the challenges of today, while refocusing on Christ, toward a successful "crossing over" journey that transcends boundaries. After redefining the concept of the mission this paper extrapolates on the importance of renewing the understanding of the mission and evangelization in a specific context affected by suffering. The methodology utilized in this study is textual analysis based particularly on the writings of theologians, researchers and practitioners in this field. This study concludes that the challenges of today force the Lebanese church "to go forth" to leave its comfort zone seeking to establish a universal solidarity that that goes beyond limits and challenges while being cemented to its own context.
\end{abstract}

Mots Cles: Mission et évangélisation. Èvangélisation. Pluralisme religieux. Laïcs. Evangelii Gaudium. Liban. 


\section{Resumo}

Este artigo explora como a comunidade cristã no Líbano recebe a missão e a evangelização no contexto do Médio Oriente. Apesar das tensões e dos desafios relacionados com as repetidas guerras e com o extremismo violento que cercam a Igreja no Líbano, a comunidade cristã é chamada a renovar o processo de profundo entendimento da sua própria missão. Os ensinamentos revelados desde a Evangelii Nunitiandi (Paulo VI) até a Evangelii Gaudium (Papa Francisco), enriquecem a experiência cristã local e redirecionam a sua missão para ser recebida no seu contexto glocal, aqui e agora! Esta missão é chamada a superar os desafios de hoje, enquanto se focam novamente em Cristo, em direção a uma jornada de "travessia" bem-sucedida que transcende fronteiras. Após se redefinir o conceito de missão, este artigo extrapola a importância de renovar o entendimento da missão e da evangelização num contexto específico afetado pelo sofrimento. A metodologia usada neste estudo é a análise textual, baseada particularmente nos estudos de teólogos, pesquisadores e profissionais nesta área. Este estudo conclui que os desafios de hoje obrigam a igreja libanesa a "ir além" da sua zona de conforto, procurando estabelecer uma solidariedade universal que ultrapasse os limites e desafios enquanto esta é baseada no seu próprio contexto.

Palavras-chave: Missão e evangelização. Evangelização. Pluralismo religioso. Não-crentes. Evangelii Gaudium. Líbano.

\section{Introduction}

"Allez donc, de toutes les nations faites des disciples" (Mt 28,19). C'est avec ces paroles que Jésus envoie ses disciples. Depuis, cet appel a toujours retenti dans les consciences chrétiennes comme un appel fondamental et fondateur de l'Église. La prédication des apôtres, le témoignage des premières communautés et, au cours des siècles, les dynamismes missionnaires aux formes multiples, manifestent la volonté permanente de l'Église de mener à bien cette tâche qui l'a marqué au plus profond d'elle-même: vivre et annoncer la Bonne Nouvelle puisque "la joie de l'Évangile remplit le cœur et toute la vie de ceux qui rencontrent Jésus". ${ }^{1}$ Cette conception confirme que l'Église

${ }^{1} \mathrm{EG} 1$. 
est missionnaire par vocation: quand elle proclame la Bonne Nouvelle, elle répond au commandement du Seigneur. La foi, l'espérance et la charité que fait naître le message, sont communicatifs. Cette conception suppose aussi que chaque croyant est appelé à participer à cette tâche missionnaire.

Le commandement solennel que Jésus laisse à ses disciples est sans ambiguité: "allez". C'est l'invitation au départ, à la rupture, au dépassement qu'opère l'accueil libre de la Parole qui est Christ. Désormais une nouvelle histoire commence comme avait commencé celle du peuple de Dieu. Les disciples sont comme un nouvel Abraham. Depuis l'envoi de Jésus, la Bonne Nouvelle de la Résurrection est vécue comme un appel à partager la joie qu'elle communique. Cette joie ${ }^{2}$ qui "remplit la vie de la communauté des disciples est une joie missionnaire"3 puisqu'elle permet aux disciples de découvrir qu'ils sont "infiniment aimé" et elle donne à l'Église son dynamisme et son identité même. ${ }^{4}$

Pour analyser les enjeux et les défis qui attendent l'Évangélisation et la mission dans notre temps, nous commencerons par définir ce que peut être l'Évangélisation aujourd'hui. Quels seront les enjeux de cette Évangélisation ? Les enjeux, c'est ce que l'on peut perdre ou gagner dans une action. Il s'agit donc de ne rien perdre de ce qui nous a été donné. ${ }^{5}$

Conscient de l'enjeu, nous n'aurons plus peur de relever les défis qui attendent les fils de Dieu dans leur annonce de l'Évangile. Nous prendrons alors pour exemple l'Église qui est au Liban. Dans une société pluri-religieuse marquée par des longues années de guerres et une récente crise économique, quels sont les défis qui attendent les Chrétiens?

\section{Limites d'une définition}

Dans son exhortation apostolique Evangelii Nuntiandi, Paul VI précise au $§ 18$ qu'évangéliser, c'est porter la Bonne Nouvelle dans tous les milieux de l'humanité et par son impact, transformer du dedans l'être humain et l'humanité toute entière:

\footnotetext{
${ }^{2}$ C'est cette joie partagée qui constitue la puissance inhérente à l'Évangile. Car comme l'écrit l'apôtre Paul dans sa lettre aux Romains, “je ne rougis pas de l'Évangile : il est une force de Dieu pour le salut de tout homme qui croit” $(\mathrm{Rm} 1,16)$.

${ }^{3}$ EG 21.

${ }^{4}$ EN 14-15; EG 4-6.

${ }^{5}$ Voir Jean 6,39: “c'est la volonté de celui qui m'a envoyé que je ne perde rien de tout ce qu'il m'a donné, mais que je le ressuscite au dernier jour".
} 
Le but de l'évangélisation est [...] bien ce changement intérieur et, s'il fallait le traduire d'un mot, le plus juste serait de dire que l'Église évangélise lorsque, par la seule puissance divine du Message qu'elle proclame, elle cherche à convertir en même temps la conscience personnelle et collective des hommes, l'activité dans laquelle ils s'engagent, la vie et le milieu concrets qui sont les leurs. ${ }^{6}$

Porter la Bonne Nouvelle, évangéliser, c'est donc proclamer un message, communiquer la Parole par laquelle Dieu se communique c'est "rendre présent dans le monde le Royaume de Dieu". ${ }^{7}$ Dieu n'est jamais sans sa Parole et sa Parole a son origine en Lui, elle est Lui. "La foi vient de ce qu'on entend", écrivait Paul dans la lettre aux Romains $10,17 .{ }^{8}$ La Bible nous révèle, à travers le témoignage des évangélistes, Jésus Christ comme Parole de Dieu faite chair. Annoncer la Parole de Dieu, c'est donc proposer l'alliance en Jésus Christ à travers le monde sans oublier la vie communautaire et l'engagement avec les autres. ${ }^{9}$ Les croyants savent que le thème de "l'alliance" désigne une réalité effective et symbolique, une réalité déjà là: il s'agit d'un magnifique échange entre le Dieu qui élit et le peuple élu comme bénéficiaire de la promesse contenue dans la Parole salvatrice. Ce thème renvoie d'ailleurs à un système d'échanges. ${ }^{10}$ Jean Rigal, dans son livre Découvrir l'Église, présente une conception renouvelée de la mission: théologale et ecclésiale. Il rattache directement l'activité missionnaire de l'Église à la mission du Dieu trinitaire, dans laquelle elle plonge ses racines et trouve son fondement. Il précise "on dépasse le seul souci de la finalité et de l'efficacité, pour approfondir l'origine et le terme de l'Évangélisation... la mission de l'Église ne nait pas d'abord d'un besoin des hommes qui seraient en attente d'un salut qui vient d'ailleurs. Elle surgit d'une nécessite intérieure à Dieu Lui-même, Celui qui, étant "communion d'amour", n'existe que dans l'action de se donner... (elle) est l'expression véritable de son être même". ${ }^{11}$

De son côté Paul Coulon ${ }^{12}$ affirme qu'il est impossible de parler et de penser "mission", sans le $\mathrm{n}^{\circ} 1$ de Lumen Gentium, dans lequel la

\footnotetext{
${ }^{6} \mathrm{EN} 18$.

${ }^{7} \mathrm{EG} 176$.

${ }^{8} \mathrm{C}$ 'est la parole entendue qui conduit à croire (EN 42).

${ }^{9}$ EG 177.

${ }^{10}$ Dieu donne la terre promise; Israël reçoit; Israël rend la terre promise en la consacrant.

${ }^{11}$ RIGAL, J., Découvrir l’Église, p. 191-192.

${ }^{12}$ COULON, P., La Mission Chrétienne De Vatican II À Aujourd'hui, p. 109.
} 
nature et le rôle de l'Église sont définis dans une formule très dense qui n'a pas cessé d'alimenter la réflexion théologique et missionnaire, comme “étant, dans le Christ, en quelque sorte, le sacrement, c'est-à-dire à la fois le signe et le moyen de l'union intime avec Dieu et de l'unité de tout le genre humain" L'important, ce ne sont pas les structures d'une Église à fonder partout dans le monde mais la réalisation du Royaume, dessein de Dieu sur l'humanité, sans distinction géographique entre pays chrétiens et pays païens.

Par ailleurs Kierkegaard ${ }^{13}$ expose un modèle de communication avec les éléments suivants: l'auteur, le message, les destinataires et le mode de communication. Ce modèle est applicable à l'Évangile. Dans le message porté par l'évangile, il s'agit d'un modèle-type de la communication où l'auteur ${ }^{14}$ est un et plusieurs, où le message renvoie à l'auteur, et où les destinataires sont préparés à recevoir le message par le message luimême. Le modèle de communication, lui, et en adéquation avec le contexte, l'environnement humain et sociologique, tout en restant respectueux du message. Kierkegaard distingue la communication directe qui transmet des informations et la communication indirecte qui initie à ce qu'il y a d'essentiel dans l'existence. Nous avons d'un côté la communication d'un savoir dont l'acquisition ne change pas l'être humain et de l'autre la communication, non seulement d'un savoir, (puisque tout message existentiel suppose une information sur l'événement), mais aussi d'un pouvoir qui transforme celui qui le reçoit. L'évangile, que nous pouvons comprendre comme faisant partie de la communication indirecte, ne peut être fermé dans un objet de savoir; c'est une communication de Dieu qui ne s'accomplit qu'à travers une appropriation personnelle de celui qui adhère.

Le message appelle à la conversion et à la participation aux promesses du Royaume. Il vise l'extrémité de la terre, donc l'universalité en niant toute forme de distinction ou de discrimination. Il a un mode de fonctionnement qui exclut tout esprit utilitaire et qui ne peut donc pénétrer le cœur de l'être

\footnotetext{
${ }^{13}$ VERGOTE, H.-B., Dialectique de la communication, introduction et traduction de deux leçons de S. Kierkegaard comme pivat-docent, p. 53-76. Voir également CLAIR, A., Pseudonymie et paradoxe, p. 305-306.

${ }^{14}$ Dans l'évangélisation, l'auteur du message est au premier degré Dieu et ensuite Jésus, qui, rempli de l'Esprit, annonce la Bonne Nouvelle. Comme porteurs inspirés de la Parole, les prophètes et les apôtres se trouvent aussi co-auteurs, parce que co-acteurs de la transmission du message. La communication du message est l'affaire de celui qui est envoyé par l'envoyé de Dieu.
} 
humain que sur le mode de la grâce. Cela signifie que Dieu se communique à travers son alliance, ses promesses et son pardon offert. ${ }^{15}$

Ainsi l'évangélisation ne peut être limitée à une communication verbale, même si cette dimension est incontournable. L'exhortation de Paul VI utilise le mot "évangélisation" comme un concept englobant toute l'activité de l'Église envoyée au monde. ${ }^{16} \mathrm{C}$ 'est dans ce sens que nous pouvons confirmer qu'entre évangélisation et promotion humaine il y a sans doute des liens profonds. Ces liens sont d'ordre anthropologiques, puisque l'être humain à évangéliser vit dans une société bien précise. Et lien d'ordre théologique, puisque nous ne pouvons pas dissocier le plan de la création du plan de la Rédemption qui atteint les situations bien précises de l'injustice à combattre et de la justice à réaliser ici-là et dès aujourd'hui.

De son côté le Pape Jean-Paul II dans son encyclique Redemptoris Missio précise que l'Église "respecte les personnes et les cultures, et elle s'arrête devant l'autel de la conscience". ${ }^{17}$ David Bosch, dans son ouvrage Dynamique de la mission chrétienne, ${ }^{18}$ ajoute que l'évangélisation est une des dimensions essentielles de la mission. Elle est la proclamation du salut en Christ à tous ceux qui ne croient pas en lui ; elle les appelle à la repentance et à la conversion, elle annonce le pardon des péchés, elle les invite à devenir des membres vivants de la communauté terrestre du Christ et à essayer de vivre au service des autres par la puissance du Saint-Esprit. Évangéliser serait dans ce sens mobiliser les gens pour le règne de Dieu, les libérer d'abord d'euxmêmes, de leur péché, de leurs chaînes, afin qu'ils vivent en hommes libres pour Dieu et leur prochain. Ainsi la "plénitude du Christ" sera recréée dans le monde, l'image de Dieu restaurée dans la vie de l'homme. ${ }^{19} \mathrm{C}$ 'est le mystère de l'Incarnation. Gaudium et spes précise "Christ dans la révélation même du

\footnotetext{
${ }^{15}$ Comme le dit si bien la DÉCLARATION de Lausanne, au § 4: "Lorsque nous transmettons l'invitation de l'Évangile, nous n'avons pas le droit de cacher ce qu'il en coûte d'être un disciple du Christ. Jésus continue d'appeler ceux qui veulent le suivre à renoncer à eux-mêmes, à se charger de leur croix et à s'identifier avec la communauté de ceux qui lui appartiennent. Evangéliser, c'est donc appeler à la mission".

${ }^{16}$ EN 6.

${ }^{17} \mathrm{RM} 39$.

${ }^{18}$ BOSCH, D. J., Dynamique de la mission chrétienne, p. 22.

${ }^{19}$ Il est nécessaire de nommer Celui que nous croyons. Les Chrétiens doivent rendre compte de l'espérance qui est en eux pour que les autres puissent reconnaître d'où leur vient cette espérance. L'apôtre Pierre écrit dans sa première lettre au chapitre 3 verset 15: "le Seigneur Christ... vous demande raison de l'espérance qui est en vous".
} 
mystère du Père et de son amour, manifeste pleinement l'homme à lui-même et lui découvre la sublimité de sa vocation... car, par son incarnation, le Fils de Dieu s'est en quelques sorte uni lui-même à tout homme". ${ }^{20} \mathrm{Il}$ faut se demander comment la théologie de l'incarnation interpelle aujourd'hui nos schémas sur la mission et l'évangélisation. Une des manières de répondre est sans doute de prendre en compte l'Église, Sacrement de Salut, dans son caractère local et inculturé. Elle est alors comme un signe qui invite à la rencontre et appelle au dialogue.

Pour le Pape Francois, évangéliser, “c'est rendre présent dans le monde le Royaume de Dieu". ${ }^{21}$ C'est "témoigner de comment on vit l'Évangile: témoigner des Béatitudes, témoigner selon l'Évangile selon saint Mathieu chapitre 25, témoigner du Bon samaritain, témoigner du pardon 70 fois de suite". ${ }^{22}$ Lors d'une rencontre avec le Conseil pontifical pour la promotion de la nouvelle évangélisation en 2014 le Pape François précise qu'il y a trois aspects essentiels pour l'évangélisation "primauté du témoignage ; urgence d'aller à la rencontre; projet pastoral centré sur l'essentiel". Il précise que "les paroles sans témoignage ne portent à rien, elles ne servent à rien ! Le témoignage est ce qui porte et donne sa valeur à la parole". ${ }^{23}$ L'évangélisation en paroles et en actes et à la lumière d'un contexte particulier, offre à chacun la possibilité et l'occasion de réorienter sa vie dans la reconnaissance en Jésus le Christ, du Sauveur et du Seigneur. Tout en gardant présentes les paroles du Pape Paul VI "aucune définition partielle et fragmentaire ne donne raison de la réalité riche, complexe et dynamique qu'est l'évangélisation, sinon au risque de l'appauvrir et même de la mutiler". ${ }^{24}$

\section{Enjeux pour une évangélisation}

Les paroles de l'Apôtre Paul : “malheur à moi si je n'annonçais pas l’Évangile” (1Cor 9,16), confirment l'envoi de Jésus. Mais elles indiquent aussi aux baptisés l'importance d'assumer leur foi en la Bonne Nouvelle qui vient offrir le salut à tout être humain sans distinction. Le Pape François

\footnotetext{
${ }^{20}$ GS 20.

${ }^{21}$ EG 176.

${ }^{22}$ L'ÉVANGÉLISATION selon le pape François, Église Catholique en Alsace, 05 dec. 2017.

${ }^{23}$ FRANÇOIS, PP., Discours aux participants à la rencontre organisée par le conseil pontifical pour la promotion de la nouvelle évangélisation.

${ }^{24} \mathrm{EN} 17$.
} 
rappel dans son exhortation apostolique Evangelii Gaudium que les chrétiens ont le devoir d'annoncer "sans exclure personne, non pas comme quelqu'un qui impose un nouveau devoir, mais bien comme quelqu'un qui partage une joie, qui indique un bel horizon, qui offre un banquet désirable. L'Église ne grandit pas par prosélytisme mais 'par attraction"'. ${ }^{25}$

Cet envoi se fait alors engagement et respect des enjeux. Nous en avons retenu cinq.

\subsection{Mondialisation et Libération}

La proclamation de l'Évangile n'est pas limitée par des frontières géographiques ou temporelles. L'appel à la conversion traverse tous les pays, toutes les cultures et tous les siècles. La mondialisation est aujourd'hui le cadre de vie. Elle se caractérise par une interdépendance des économies. Elle se traduit par une concurrence accrue sur les marchés et une mobilité internationale plus grande des hommes, du travail et du capital. C'est la présence du monde entier dans le quotidien.

L'Église est témoin de la transnationalisation des entreprises. Plusieurs de ces entreprises ont une puissance telle que face à elles, des gouvernements perdent une large part de leur liberté d'action, voire de leur souveraineté économique, politique, culturelle, judiciaire ou surtout sociale. Tout en invoquant la liberté du commerce, certaines entreprises imposent en effet des règles qui tiennent trop peu ou pas du tout compte de l'individu, du bien commun ou des entreprises de moindre envergure. ${ }^{26}$ Sous le couvert du libreéchange, nous voilà tombés sous la coupe d'un réel péril de course aveugle au profit. La rentabilité devient l'unique horizon du commerce. L'absolutisation de l'efficacité crée de l'exclusion. Et la mondialisation est capable aussi de générer des exclus à toutes les échelles. Ainsi l'Église assiste à une définition d'un principe d'exclusion qui légitime et justifie les attaques contre la vie humaine. ${ }^{27}$

\footnotetext{
${ }^{25}$ EG 14.

${ }^{26}$ Les patriarches catholiques d'Orient écrivent dans leur lettre de Pâques 1992: "nous espérons que le dernier mot sera pour le bien commun et que seront consolidés les fondements de la justice, de la paix et du développement pour tous les hommes". La présence chrétienne en Orient, Mission et témoignage adressé à leurs fidèles, en Orient et dans la diaspora, 6 .

${ }^{27}$ Les paroles de Centesimus Annus se font alors toujours d'actualité: "Avant même la logique des échanges à parité et des formes de la justice qui les régissent, il y a un certain dû à l'homme parce qu'il est homme, en raison de son éminente dignité" (CA 34).
} 
La Mondialisation apporte un questionnement nouveau sur la vision de l'homme. Dans l'état actuel des choses, la Mondialisation est un mouvement incontournable mais il ne faut pas qu'elle soit incontrôlable. Il faut donc l'accueillir avec ouverture, lucidité mais aussi détermination.

L'activité missionnaire implique dès le début et de toute évidence que les croyants deviennent sensibles aux besoins des autres, qu'ils ouvrent les yeux et s'engagent pour l'homme. ${ }^{28}$ Dans ce contexte, les Chrétiens doivent rendre la Parole de Dieu accessible. Plusieurs aréopages ${ }^{29}$ attendent l'implication du croyant-envoyé pour venir au secours de l'homme. Evangelii Nuntiandi § 30 précise que "L'Église... a le devoir d'annoncer la libération". ${ }^{30} \mathrm{Il}$ faut tenir à la fois l'annonce du Règne et la proclamation des libérations humaines. ${ }^{31}$ L'un ne remplace pas l'autre... et inversement.

\subsection{Les principales difficultés qui attendent l'évangélisation}

L'évangélisation en Europe doit de plus en plus faire face à une situation d'absence de Dieu. Même dans les régions restées très chrétiennes, voire dans le cœur de chaque croyant, l'athéisme -théorique ou pratique - laisse son empreinte. Nous observons aussi un phénomène "d'indifférence religieuse contemporaine", telle que la nomme Maurice Vidal dans son article "la "nouvelle évangélisation". ${ }^{32}$ Déjà en 1999 Hippolyte SIMON, dans son livre Vers une France Païenne ? note l'importance du retour d'un paganisme. ${ }^{33} \mathrm{Il}$ précise:

\footnotetext{
${ }^{28}$ L'espoir d'une nouvelle évangélisation est de favoriser, selon l'expression de Jean-Paul II, une "nouvelle synthèse créatrice entre l'Évangile et la vie".

${ }^{29}$ Paul, après avoir prêché dans de nombreux endroits, parvient à Athènes et se rend à l'Aréopage, sur l'Agora où il annonce l'Evangile en utilisant un langage adapté et compréhensible dans ce milieu (At 17,22-31). L'Aréopage représentait alors le centre de la culture des Athéniens instruits et il peut aujourd'hui être pris comme symbole des nouveaux milieux où l'on doit proclamer l'Evangile. Jean-Paul II écrit dans Redemptoris Missio, 37: "Il existe, dans le monde moderne, beaucoup... d'aréopages vers lesquels il faut orienter l'activité missionnaire de l'Église. Par exemple, l'engagement pour la paix, le développement et la libération des peuples, les droits de l'homme et des peuples, surtout ceux des minorités, la promotion de la femme et de l'enfant, la sauvegarde de la création, autant de domaines à éclairer par la lumière de l'Evangile".

${ }^{30}$ EN 30.

${ }^{31} \mathrm{EN} 34$.

32 VIDAL, M., La nouvelle évangélisation, p. 42.

${ }^{33}$ Il écrit: "devenus amnésiques de la Parole qui les a faits, nos contemporains, dans leur majorité, retournent aux idoles muettes du paganisme. Autant dire à l'esclavage de la fatalité". SIMON, H., Vers une France païenne?, p. 69.
} 
Si j'ai suggéré, pour [...] décrire [la société française], l'adjectif "païen", au sens qu'il a dans les lettres de Saint Paul, ce n'est pas pour nous décourager mais pour inviter à proposer la foi. Il est important de savoir quelle est la religion dominante aujourd'hui. On le voit bien dans les Actes des apôtres : Paul ne parle pas de la même façon aux juifs, dans les synagogues, ou aux païens, sur l'Agora, à Athènes. ${ }^{34}$

Face à ce genre de situation, au vide spirituel, beaucoup de maîtres de pensée sont prêts à apporter leur thérapie pour guérir le mal de la société. Ce sont entre autres les sectes, dont le foisonnement a surpris tous les observateurs ces dernières années.

Vient s'ajouter à cela le développement d'un réflexe individualiste habité par une recherche fébrile de son redéploiement personnel, hostile finalement à une vraie ouverture et au dépassement de soi-même. L'individualisme renferme chacun dans son propre système de références.

Au Liban, comme en Syrie ou en Égypte par exemple, nous ne pouvons pas parler "d'indifférence religieuse", mais plutôt "d'implication religieuse intéressée". L'appartenance religieuse, revendiquée, se limite souvent à un cadre ou une représentation sociale. Le pluralisme religieux se limite alors à une simple cohabitation. Il peut même devenir violent quand la peur et l'extrémisme s'installent. ${ }^{35}$ La guerre en Syrie, les conflits et les guerres répétées au Liban, les mutations dont l'Égypte a survécu récemment ne font que signaler un sérieux malaise à ce niveau.

\subsection{Rien ne remplacera l'expérience libre et personnelle de la foi}

L'Évangélisation est toujours une invitation qui porte un message d'espérance au monde pour communiquer la liberté et la joie à chacun. Elle invite à reconnaître et à accepter, par une réponse et une décision personnelle, le Christ fils de Dieu mort et ressuscité pour tous. Or qui dit réponse et décision personnelle dit en même temps liberté. N'oublions pas que la conversion à laquelle le Christ appelle passe par la vie de l'homme: "elle invite à des transformations concrètes", comme le précise l'affirmation œcuménique La Mission et l'Évangélisation ${ }^{36}$ au paragraphe 11. Parlant de la

\footnotetext{
${ }^{34}$ SIMON, H., "L’Église naît de l'avenir...", p. 7.

${ }^{35}$ Souvent sur le seul critère de l'image que l'on a de l'autre depuis des siècles, et dans lequel on l'a enfermé.

${ }^{36}$ Document du Conseil œcuménique des Églises, 1990.
} 
conversion des non-Chrétiens, les pères de Vatican II écrivent dans le décret Ad Gentes: "le Saint-Esprit ouvrant leur cœur (Act. 16,14) [ils] croient et se convertissent librement au Seigneur". ${ }^{37}$ Dans ce sens, nous pouvons affirmer que la mission n'est pas d'abord un faire, mais un don à recevoir. Avant d'être notre œuvre, la mission est l'œuvre de Dieu. Avant d'être une annonce, elle est une conversion. L'évangélisation n'est pas le fruit de la peur, de la menace ou d'un sentiment de culpabilité, mais le fruit de l'amour. Elle est aussi une recherche permanente de la croissance,

Ce qui implique de prendre très au sérieux chaque personne et le projet que le Seigneur a sur elle. Chaque être humain a toujours plus besoin du Christ, et l'évangélisation ne devrait pas accepter que quelqu'un se contente de peu, mais qu'il puisse dire pleinement: 'Ce n'est plus moi qui vis, mais le Christ qui vit en moi' $(\mathrm{Ga} 2,20) .^{38}$

\subsection{Une évangélisation jamais achevée}

Où peut-on observer que l'évangélisation telle que nous l'avons définie est déjà achevée? Où peut-on affirmer que la Bonne Nouvelle est complètement reçue? Les guerres, l'extrémisme violent, la corruption, l'esclavage moderne, la pauvreté etc... nous laissent comprendre assez vite que l'évangélisation est toujours d'actualité. La Bonne Nouvelle ne se répand pas de la même manière que se forme une nation ou un groupe. Elle se réalise par une prédication toujours à reprendre, et par un accueil de l'évangile toujours à remettre en cause. La nouvelle évangélisation n'est pas pour nous une restauration d'un paradis perdu, d'une société imaginée comme pleinement accordée à l'Évangile en un temps idéalisé. Les défis que la première communauté chrétienne a confrontés n'étaient pas du tout facile. Par conséquent, "ne disons pas qu'aujourd'hui c'est plus difficile; c'est différent". ${ }^{39}$ L'évangélisation est rencontre entre la nouveauté de l'Évangile et la nouveauté de la culture humaine, celle d'aujourd'hui et celle de demain. Il n'est pas étonnant que cette rencontre puisse provoquer une confrontation avec le monde ${ }^{40}$ car l'Église n'a

\footnotetext{
${ }^{37}$ AG 13.

${ }^{38}$ EG 160.

${ }^{39}$ EG 263.

${ }^{40}$ Elle doit prendre toujours la défense de la dignité humaine. Il ne s'agit pas pour autant de s'enfermer dans une fonction du juge, mais de repérer les énergies disponibles dans la société, afin de les raviver et leur donner une force libératrice. Elle doit donc favoriser tout ce qui fait
} 
pas à s'approprier aveuglément une culture. Le Pape François nous le rappel dans son Exhortation Apostolique

Chaque jour, dans le monde renaît la beauté, qui ressuscite transformée par les drames de l'histoire. Les valeurs tendent toujours à réapparaître sous de nouvelles formes, et de fait, l'être humain renaît souvent de situations qui semblent irréversibles. C'est la force de la résurrection et tout évangélisateur est un instrument de ce dynamisme. ${ }^{41}$

Nous savons fort bien que la foi chrétienne n'est pas héréditaire. Elle n'est pas acquise une fois pour toutes; elle est à renouveler en permanence, à approfondir et à lire en fonction de sa vie aujourd'hui. Elle naît et vit grâce à la Parole de Dieu que l'Esprit fait entendre et grâce à l'événement de témoignage. Mais elle doit parfois être réveillée par une nouvelle évangélisation. Soyons clairs, nous distinguons donc entre "évangélisation" et “nouvelle évangélisation". L'évangélisation est la proclamation de la Parole là où elle est encore inconnue, tandis que la nouvelle évangélisation, à l'opposé de la première, part d'un athéisme ou d'un agnosticisme, d'un refus conscient de l'alliance que Dieu propose. Elle suppose que la Parole a déjà été annoncée. Ne nous leurrons pas: la Parole peut-elle être complètement reçue? Sans doute pas! D'où l'importance de parler d'une évangélisation qui est et qui restera toujours à reprendre ${ }^{42}$ ou à enraciner pour reprendre le mot que nous avons employé en parlant de l'exhortation selon Matthieu. Cette nouvelle évangélisation serait selon les paroles du Pape François faite avec "l'Esprit Saint, parce qu'il est l'âme de l'Église évangélisatrice" elle serait incarné à travers "un fort engagement social et missionnaire, ... une spiritualité qui transforme le cœur". ${ }^{43}$

\footnotetext{
jaillir la vie en prenant le risque de dévoiler tout ce qu'une "culture de mort et d'esclavage" risque de désagréger dans la vie sociale et dans la vie de chacun.

${ }^{41}$ EG 276.

${ }^{42}$ De son côté, WIDMER, G., Quelques repères d'une théologie de l'évangélisation, p. 384, distingue la pré évangélisation et l'évangélisation à proprement parler. Il écrit: "comme première phase de la tâche missionnaire de l'Église, la pré évangélisation (l'antique praeparatio evangelii) vise à faire rencontrer personnellement le Christ à celui qui l'a oublié ou qui l'ignore, en les sensibilisant au sens de leur existence à partir de leur situation concrète. Les deux phases suivantes, celles de l'évangélisation, comprennent la proclamation du Christ, sauveur et seigneur, et l'ppel à le suivre, puis l'initiation catéchétique au baptême".

${ }^{43}$ EG 262.
} 
Jésus "le tout premier et le plus grand évangélisateur" 44 fonde par sa Résurrection ${ }^{45}$ la certitude que l'événement du Salut est donné aujourd'hui. Le Christ eucharistique nous le rappelle sans cesse. Les quatre évangélistes et Paul (1Cor 9,1; 15,6-11; 2Cor 4,6-13; G1 1,15-16) sont d'accord pour affirmer que c'est bien la Résurrection du Christ qui est le point de départ de la mission. Les témoins que Christ envoie portent en eux le Christ Ressuscité. Bultmann écrit ainsi: "Christ est ressuscité dans le kérygme de l'Église". ${ }^{46}$

Le message de la résurrection porte aux moins deux points majeurs qui s'imposent dans le travail d'évangélisation:

- d'abord la résurrection signifie et réalise en effet la délivrance des mutilations et des ténèbres dues aux péchés, sous toutes leurs formes: nous citons parmi d'autres les divisions, l'esclavage, l'extrémisme, les guerres et l'individualisme qui écrasent plusieurs sociétés... Or le Christ ressuscité "sauve" au sens radical du mot, il attire le croyant dans la vie du Royaume et l'entraîne dans le mystère pascal.

- ensuite comme Christ est ressuscité, il vit, il est toujours vivant. Son évangile devient alors un présent éternel dans l'histoire. Parce qu'il est ressuscité au terme de sa mission salvatrice, c'est l'événement tout entier de son intervention dans l'histoire qui échappe à la limitation d'un passé révolu ${ }^{47}$ et qui inaugure définitivement son règne.

\footnotetext{
${ }^{44} \mathrm{EG} 12 ; \mathrm{EN} 7$.

45 'La croix que l'Église proclame juge aussi l'Église et censure toute manifestation de complaisance à propos de ses 'succès'. Une Église qui s'autocongratule contrecarre la puissance de la croix dans sa vie et son ministère. La croix ne porte pas un message de jugement mais aussi de pardon et d'espérance, et cela est vrai pour l'Église également'. BOSCH, D. J., Dynamique de la mission chrétienne, p. 522

${ }^{46}$ La résurrection de Jésus comme telle échappe à l'histoire au sens commun du terme: elle est entrée dans l'histoire non seulement par l'apparition, mais par la prédication des apôtres et la foi en leur parole. Et c'est dans cette parole qu'il se manifeste comme le Christ ressuscité et glorifié. La propagation de l'Evangile, vécue dans la certitude d'une mission reçue, et l'accueil de l'Evangile, compris comme naissance à une vie renouvelée, sont autant que l'apparition, des signes de l'irruption de Jésus, après sa mort, dans l'histoire et l'expérience des hommes. DELORME, J., La Résurrection de Jésus dans le langage du Nouveau Testament, p. 165.

${ }^{47}$ On comprend dès lors la place centrale occupée par le Christ ressuscité, tant dans le Kérygme apostolique que dans l'enseignement de Paul: "Et si le Christ n'est pas ressuscité, vaine est votre foi, vous êtes encore dans vos péchés" (1Cor 15,17).
} 
Nous voyons donc dans l'évangélisation l'importance de renouer, non avec un concept ou un programme mais avec une personne qui a le visage et le nom de Jésus, mort et ressuscité, vivant et présent aujourd'hui pour l'ensemble de l'humanité. Il semble alors nécessaire à l'Église d'aujourd'hui de vérifier continuellement si sa façon de comprendre le Christ correspond à celle des premiers témoins. C'est l'auto-communication de Dieu en Christ qui précède et conditionne la proclamation de la Bonne Nouvelle. Lucien Legrand précise dans son livre Le Dieu qui vient: "La présence messianique ne se subordonne pas à la mission: elle la domine. Elle constitue le milieu dans lequel la mission va s'exercer". ${ }^{48}$

\section{Défis pour aujourd'hui et pour demain: perspectives libanaises}

Le Pape Jean-Paul II nous rappel dans sa Lettre Encyclique Redemptoris missio que l'activité missionnaire "représente, aujourd'hui encore, le plus grand des défis pour l'Église". Pour analyser cela nous développons dans cette partie les défis qui attendent l'Église au Liban. Nous n'avons pas l'objectif d'isoler l'Église du Liban de l'Église universelle ni d'en faire un modèle. Mais nous voulons étudier la théologie de l'incarnation et penser l'évangélisation en prenant en compte l'Église en un lieu, dans un milieu. Nous avons choisi le Liban.

\subsection{Développer une pastorale Prophétique}

Par sa mission, l'Église, au Liban ou ailleurs, est appelée à être un "signe" prophétique qui permette de mettre en œuvre la transformation du monde. Or ne l'oublions pas, l'Église reçoit sa Mission du Ressuscité. Il en est le centre et le fondement. Envoyée par Lui, l'Église libanaise est appelée à aller aux frontières de la société, sans avoir peur de se perdre dans le monde. Cela nécessite un certain équilibre à trouver et à assumer. L'Église ne peut être missionnaire que si son être-dans-le-monde est en même temps un êtredifférent-du-monde. ${ }^{49}$ "L'évangélisation (...) ne peut pas ne pas contenir l'annonce prophétique d'un au-delà", écrivait Paul VI au paragraphe 28 de Evangelii Nuntiandi.

${ }^{48}$ LEGRAND, L., Le Dieu qui vient, p. 110.

${ }^{49}$ HENDRIKUS, B., Christian Faith, p. 415. 
Être un signe prophétique oblige l'Église à vivre de la "charité, source et critère de la mission... l'unique critère selon lequel tout doit être fait, ou ne pas être fait". ${ }^{50}$ Or la charité est une grâce qui s'exprime et s'accomplit à travers l'amour du prochain. Le prochain est pour l'Église le moyen unique d'incarner la charité. Il permet la rencontre de Dieu (Mt 25). La charité vécue permet à celle qui est envoyée aux frontières de la société d'incarner l'esprit de la Bonne Nouvelle dans le monde en tout temps et surtout pendant les moments difficiles marqués par la guerre et la haine. Universelle par définition, la charité passe par un dépassement de soi-même. Elle brise le mur de la peur qui handicape la rencontre avec l'autre. La pastorale prophétique affronte les phénomènes de la société à la lumière du salut promis par le Christ sauveur. Par sa mission prophétique, l'Église du Liban est invitée à dénoncer tout ce qui va à l'encontre de l'être humain et fait obstacle à la communion des hommes entre eux et avec Dieu venu apporter le Salut.

Mais cette mission prophétique exige de la part de l'Église une attitude profonde de pauvreté, de dépouillement, donc de rupture; chaque Église locale pourrait aussi voir ce qui l'empêche de rencontrer l'autre. De quoi a-t-elle besoin de se dépouiller? Qu'est-ce qui retarde sa réalisation en tant que témoin à son tour fidèle? A-t-on réellement besoin de tant de richesses et de pouvoirs au moment où l'essentiel est Un? "Évangélisatrice, l'Église commence par s'évangéliser elle-même" écrivait Paul VI dans Evangelii Nuntiandi au § $15 .{ }^{51}$

La pastorale prophétique doit avant tout être porteuse de témoignage car "l'homme contemporain croit plus les témoins que les maîtres", écrivait Jean-Paul II dans Redemptoris missio au $\S 42$. Elle peut en tout cas prendre plusieurs formes. Nous allons maintenant étudier le point le plus urgent pour nous: Où et comment l'Église du Liban, inspirée par l'esprit prophétique, doitelle intervenir et prendre des initiatives?

\subsubsection{Servir l'ensemble de la société}

“L'évangélisation du monde n'est pas avant tout une affaire de paroles ou d'activités, mais de présence: présence du peuple de Dieu au milieu de l'humanité, présence de Dieu au sein de son peuple". ${ }^{52}$ Une des pistes pourrait

\footnotetext{
${ }^{50} \mathrm{RM} 60$.

${ }^{51}$ L'Église ne fait qu'ouvrir à une transcendance: 1'homme, déjà habité par Dieu, Le cherche ou ne Le cherche pas dans son existence.

${ }^{52}$ MARTIN-ACHARD, R., Israël et les nations, p. 72.
} 
être de rendre l'Église présente dans les différents groupements humains vers lesquels les témoins sont envoyés. Il s'agirait d'être présent non pour s'approprier un message ou garantir des privilèges, mais plutôt pour rendre témoignage. ${ }^{53}$ Comment réussir le passage d'une présence passive à une présence engagée, habitée par un esprit de charité et par l'espérance annoncée par le Christ malgré les souffrances des guerres et de leurs conséquences? La "missiologie du chemin", chère au théologien David BOSCH, attend toutes les communautés présentes au Liban.

Être présent à l'autre, mais aussi, chaque fois que l'autre l'accepte, l'Église doit se mettre à son service. Le Christ ne s'est pas contenté de se dire serviteur, mais il a appelé ses disciples à suivre ses traces et à être un signe de sa présence au milieu des hommes en servant autrui: “C'est un exemple que je vous ai donné, pour que vous fassiez, vous aussi, comme moi j'ai fait pour vous" (Jn 13,15).

La mission de l'Église, c'est le service de l'Évangile. Comme nous l'avons signalé plus haut la mission doit tendre à l'annonce explicite de JésusChrist en gardant son sens de manifestation de l'amour de Dieu et incarnation de l'Évangile dans le monde. ${ }^{54}$

L'engagement de tous les citoyens est nécessaire à l'édification de la cité. Le temps presse et l'occasion est venue de participer à la construction de la société dans tous les domaines. Or personne ne doit être exclu ou marginalisé à cause de son appartenance religieuse ou pour toute autre raison. ${ }^{55}$

53 L'assemblée des patriarches catholiques d'Orient écrit dans sa lettre pastorale La présence chrétienne en Orient, mission et témoignage adressés à leurs fidèles, en Orient et dans la diaspora, Pâques 1992 : "La présence signifie que nous sommes, au milieu de la société où nous vivons, un signe de la présence de Dieu dans notre monde. Cela nous invite à être 'avec', 'dans', 'pour' et non pas 'contre', 'en dehors' ou encore 'en marge' de la société où nous vivons... Notre présence chrétienne ne peut et ne veut pas être une présence pour nous-mêmes. Car le Christ n'a pas fondé son Église pour qu'elle soit au service d'elle-même, mais pour qu'elle soit confessante et porteuse d'une mission, la mission même de son fondateur et Maître" (17-18). Nécessaire, cette présence-là n'est pas suffisante pour autant. Cet "être-avec" le monde de l'Église ne peut se réduire à un "être-comme" le monde.

${ }^{54} \mathrm{Au}$ paragraphe 5 du décret sur l'apostolat des laïcs (AA), les pères de Vatican II écrivent: "la mission de l'Église (...) n'est pas seulement d'apporter aux hommes le message du Christ et sa grâce, mais aussi de pénétrer et de parfaire par l'esprit évangélique l'ordre temporel".

${ }^{55} \mathrm{C}$ 'est ainsi que nous recevons les paroles-programmes des patriarches catholiques d'Orient lorsqu'ils écrivent: "nous voudrions que toute la société sache (...) opposer une résistance qui bloque tout fanatisme ou extrémisme. Toutes les institutions sociales et religieuses doivent collaborer pour extirper ce phénomène, par un plan éducatif général et une action constante animée d'un esprit d'amitié" (AA 23). 


\subsubsection{Jamais sans les laïcs}

La pastorale prophétique de l'Église ne peut prendre forme qu'à travers des engagements concrets. Il nous semble essentiel que l'Église libanaise intègre des laïcs dans sa mission d'évangélisation. La centralisation de la mission autour des seuls ministres ordonnés, telle que nous l'observons dans l'Église libanaise, ne peut que retarder la réalisation de l'Église-peuple de Dieu, où clercs et laïcs sont ensemble en coresponsabilité pour servir la Bonne Nouvelle. L'annonce de l'Évangile ne peut rester l'affaire des seuls "spécialistes". Elle incombe à tout Chrétien conscient de sa vocation de baptisé. ${ }^{56}$ Elle appel l'Église à s'impliquer plus dans la "formation des laïcs et l'évangélisation des catégories professionnelles et intellectuelles". ${ }^{57}$

L'Église du Liban devra développer une ecclésiologie où les laïcs, hommes et femmes ${ }^{58}$ sont considérés en partenaires à part entière. Ils doivent participer de manière active et responsable à la vie ecclésiale, au sein des différentes structures et des divers conseils, à la mesure de leurs compétences. Mais rien ne se fera sans la confiance et sans l'intégration de l'esprit de communion.

\section{2. Évangélisation et souffrance}

L'évangéliste Luc souligne que la mission va nécessairement au-devant de l'adversité et de la souffrance. Il retrace le voyage de Jésus de la Galilée à Jérusalem (Lc 9,51-19,40) en montrant à plusieurs reprises que c'est un parcours vers la passion et vers la mort. ${ }^{59}$ Or ce qui vaut pour le maitre vaut aussi pour les disciples. Avec l'évangéliste Marc (8,34-38), Luc partage bien des propos sur la souffrance à venir des disciples, mais aux paroles de Jésus invitant à "prendre sa croix", Luc ajoute "chaque jour" $(9,23)$.

La guerre au Liban et la récente crise économique et la pandémie du Corona qui a frappé l'ensemble de la population et l'explosion du Port de Beyrouth qui a détruit une immense partie de la capitale n'ont fait qu'amplifier les souffrances, rendant le message de paix de plus en plus urgent. La situation actuelle, au Liban et dans le Moyen-Orient, les difficultés économiques qui

\footnotetext{
${ }^{56} \mathrm{AG} 32$.

${ }^{57} \mathrm{EN} 102$.

${ }^{58}$ EG 103-104.

${ }^{59}$ Plusieurs propos le soulignent: Luc 9,51; 13,33; 17,25; 18,31-34; $24,7$.
} 
paralysent le pays et enfonce le Liban dans une incertitude financière ne permettent pas de dire que le chemin sera facile et le fardeau léger (Mt 11,29). Pour mieux expliquer l'évangélisation dans les temps de souffrance, nous développerons notre pensée dans les points suivants.

\subsubsection{Le chômage et l'émigration}

Parmi les souffrances, nous soulignons celles qui font ravage chez tous les enfants du pays: le chômage ${ }^{60}$ et l'émigration. L'Église du Liban, comme celle d'Orient, souffre de ce phénomène d'une façon encore plus particulière puisque les fidèles n'ont pas cessé d'immigrer, et cela depuis plusieurs siècles. Face à ces souffrances, l'Église est appelée à ne pas rester passive. En l'absence d'un projet social national qui puisse résoudre le mal du pays, l'Église est appelée à venir au secours de la jeunesse du pays avant qu'il ne soit trop tard. Comment continuer à témoigner autrement dans un contexte marqué par le couple infernal: chômage et émigration? Jusqu'où la promesse de salut promise par le Christ peut-elle être incarnée dans la vie d'une Église dont le seul espoir de ses fidèles est d'immigrer?

\subsubsection{Les personnes touchées par la pauvreté et la crise économique}

La pauvreté se creuse en l'être humain de multiples manières. Sommairement, nous retenons la pauvreté de biens économiques, de pauvreté physique ou psychique, de pauvreté relationnelle et de pauvreté intellectuelle. Autant d'aspects qui se traduisent concrètement en termes d'oppressions car d'une façon générale, "le pauvre est d'abord l'opprimé, celui qui est mis au dernier rang de la société, qui est méprisé". ${ }^{61}$

La réalité de la crise économique qui a suivi les longues années de guerres a réduit de plus en plus de personnes à un état de pauvreté au Liban. Une pénurie réelle est observée, le plus souvent impossible à surmonter par les seuls efforts des victimes et encore moins par des pétitions de principe. Face à cette réalité sociale douloureuse et à ses conséquences anthropologiques, le message d'évangélisation est aussi attendu et "nous devons dire non à une

\footnotetext{
${ }^{60}$ Juste pendant la période d'Octobre jusqu'à Février 2020 plus de 785 restaurants ont fermés leurs portes et plus de 25.000 personnes ont perdus leur travail.

${ }^{61}$ TRILLING, W., Pauvreté, p. 371-375.
} 
économie de l'exclusion et de la disparité sociale"62 car et comme le rappel le Pape François tant que ne s'éliminent pas "l'exclusion sociale et la disparité sociale il sera impossible d'éradiquer la violence". ${ }^{63}$ En plus de l'importante œuvre culturelle, scolaire et universitaire que l'Église libanaise assure elle peut encore intervenir au moins à deux niveaux. Elle est capable:

- d'annoncer le message de salut et de libération humaine promise par le Christ; de libérer l'être humain réduit à une humanité bafouée.

- de créer ou de soutenir des projets d'intégration qui mettent un terme à leur souffrance. Cela signifie que les Églises peuvent aussi user de leurs propres moyens, biens et richesses, pour venir au secours des personnes les plus démunies.

\subsection{Réussir le pluralisme religieux par le dialogue}

Un prédicateur occidental, animant une conférence à Beyrouth dans les années 80, commença sa conférence ainsi: “Depuis combien d'années les Maronites vivent-ils avec les Druzes?" Un des participants lui répondit: "Depuis plus de 800 ans". Le conférencier réagit en demandant: "Et ils sont encore Druzes?"

Ces paroles peuvent révéler une certaine forme d'attentes du pluralisme religieux. Pour certains, il ne peut réussir qu'en conduisant à la conversion de l'autre. Mais peut-être que les paroles de ce conférencier signifient qu'aucune rencontre avec l'autre ne peut nous laisser indifférent. Sûrement ces Druzes, en 800 ans, ont dû faire un cheminement dans le temps. Mais les Chrétiens l'ont fait aussi. Nous pouvons même poser la question suivante: ces Chrétiens sontils toujours chrétiens? Le sont-ils comme s'ils n'avaient jamais été confrontés à d'autres croyants? Sûrement pas.

"L'Évangélisation implique aussi le dialogue" ${ }^{44}$ et pour réussir le pluralisme religieux dans ce sens il ne s'agit pas de gommer les difficultés et les tensions qui ont marqué des siècles de coexistence, il consiste plutôt à le faire vivre. Le dialogue inter-religieux "porte l'Église à maintenir et à intensifier les relations avec les non chrétiens" ${ }^{\circ 5}$ peut permettre à l'ensemble

\footnotetext{
${ }^{62}$ EG 53.

${ }^{63}$ EG 59.

${ }^{64} \mathrm{EG} 238$.

${ }^{65} \mathrm{EG} 251$.
} 
des communautés d'assumer leur héritage propre, de vivre ensemble et de faire approfondir leur propre perception de la foi ${ }^{66}$ Le dialogue implique la rencontre de l'interlocuteur, le respect face à sa liberté et la découverte des éléments de vérité contenus dans les vues de l'autre il s'agit d'une "attitude d'ouverture en vérité et dans l'amour". ${ }^{67}$ Dans le dialogue, les interlocuteurs sont invités les uns les autres à s'engager sur un chemin de conversion ${ }^{68}$ puisque "nous pérégrinons ensemble". ${ }^{69}$ Non pas une conversion au sens commun du terme, mais au sens biblique c'est à dire le retour d'un cœur humble à Dieu. La mission et le dialogue comportent dans ce sens le respect de l'autre, fondé sur la proclamation de la Bonne Nouvelle de Jésus-Christ, en reconnaissant et en encourageant la liberté religieuse et l'engagement en faveur de l'impératif missionnaire. ${ }^{70}$

Au Liban aujourd'hui, le dialogue apparait comme une clef pour le dépassement des limites individuelles et communautaires. Les patriarches catholiques écrivent: "L'Orient est la terre du dialogue entre l'homme, tout au long de l'Économie du Salut. Ce dialogue atteint son point culminant dans le Christ". ${ }^{71}$ Le dialogue est l'établissement d'un moyen efficace pour établir une vraie communion entre les personnes, les communautés et Dieu. Le dialogue brise le mur des préjugés nourris par les haines et les incompréhensions fratricides. Il n'est pas une réalité passive au cours de laquelle chaque interlocuteur se contenterait de recevoir des informations sur ce que vit et pense son partenaire. Mais il prend sa place dans la découverte, par les deux parties, du don multiforme de Dieu à l'Homme (Leonard Swidler, 2012). Il ouvre les hommes et les différentes communautés les uns aux autres, il les met en mesure de s'aider mutuellement ${ }^{72}$ à réaliser leur vocation propre. ${ }^{73}$

\footnotetext{
${ }^{66}$ SWIDLER, L., Deep-Dialogue/Critical-Thinking/Competitive-Cooperation, pp 143-151. ${ }^{67} \mathrm{EG} 250$.

${ }^{68}$ Nous lisons dans le document "orientations concernant le dialogue et la mission", publié en 1984, que "dans le langage biblique chrétien, la conversion est le retour d'un cœur humble et contrit à Dieu, avec le désir de lui soumettre plus pleinement sa propre vie. A cette conversion tous sont constamment invités". (Dialogue et la Mission, 37).

${ }^{69}$ EG 244.

70 CONGREGATION POUR L'EVANGELISATION DES PEUPLES, L'Eglise du Christ en Mission dans le Monde, p. 389-390.

${ }^{71}$ PATRIARCHES CATHOLIQUE D'ORIENT, La présence chrétienne en Orient, Mission et témoignage adressé à leurs fidèles, en Orient et dans la diaspora.

72 SWIDLER, L., Understading Dialogue, p. 9-24.

${ }^{73}$ Une nouvelle attitude s'impose: celle du dialogue et de la réciprocité. Le mot "dialogue" ponctue l'encyclique Ecclesiam suam, la première de Paul VI (06/08/1964), et l'on entendra
} 
Maurice Pivot précise "Il y a dialogue interreligieux au sens fort du terme lorsque chaque partenaire se laisse interroger au cœur même de sa foi et de son expérience spirituelle et sort transformé par la rencontre". ${ }^{74}$ Il prend place dans les efforts de l'être humain pour accueillir le Royaume de Dieu en lui. Il a donc une réelle dimension missionnaire, sans viser directement le passage de l'interlocuteur d'une tradition religieuse à une autre. ${ }^{75}$

Le dialogue interreligieux peut se situer au moins sur deux niveaux: le dialogue entre les responsables communautaires et le dialogue et les initiatives lancés au niveau des fidèles. Il vise à éliminer les préjugés et les fausses images de l'autre et il engage les partenaires dans des gestes communs qui apportent la contribution spécifique des religions à la réconciliation nationale et à la paix. Il ouvre le chemin vers le dialogue des personnes. Dans son article intitulé "Vivre la mission de l'Église dans la maison de l'Islam", Henri Tessier écrit que "le dialogue religieux entre les systèmes a pour but d'assurer une meilleure connaissance réciproque... de chercher les voies d'un engagement concret pour la réconciliation et la construction d'une société plus humaine". Il affirme que "ce travail est à peine commencé entre chrétiens et musulmans, du moins dans le monde arabe. Sauf exception, rien ne permet de penser qu'il existe une vraie volonté de comprendre l'autre dans sa spécificité et de l'accepter tel qu'il est". ${ }^{76}$ Le vivre-ensemble promeut alors le témoignage des bienfaits du dialogue pour les communautés. Le travail sur le terrain, au service de la Paix, contre la misère ou la violence, pour faire grandir l'être humain dans sa dignité et peut aussi être un espace de rencontre et de témoignage.

\section{Conclusion}

Pour conclure, nous voudrions souligner l'importance de la vérité du témoignage qui peut être vécu par les disciples d'aujourd'hui. Le message chrétien a été convaincant car il était un message habité par ceux qui le proclamaient et vécu communautairement (Actes 2,42) par les apôtres et

longtemps résonner ces formules percutantes: "l’Église se fait parole; l’Église se fait message; l'Église se fait conversion" (ES 67). Et c'est encore dans sa première encyclique (1979) que Jean-Paul II aura ce mot expressif: "l'attitude missionnaire commence toujours par un sentiment de profonde estime face à ce qu'il y a en tout homme" (RH 12).

${ }^{74}$ PIVOT, M., Un nouveau souffle pour la mission, p. 41.

75 “C'est le Saint-Esprit qui guide la réalisation du projet de Dieu dans l'histoire des individus et du genre humain" (Dialogue et Mission, 43).

${ }^{76}$ TESSIER, H. M., Vivre la mission de l'Église dans la maison de l'Islam, p. 40. 
les missionnaires; c'était un message efficace qui mettait en question les habitudes et les acquis installés par l'histoire et la tradition; il a permis des dépassements et des ruptures. Mais il était aussi un message nouveau, ancré en Dieu venu libérer l'homme. Or cette nouveauté est de plus en plus pertinente dans un millénaire marqué par l'extrémisme violent qui limite la perception authentique de tout autre. Il permettrait de structurer l'existence autour d'une personne, Jésus-Christ.

L'extrémisme et la souffrance qui affectent le monde d'aujourd'hui oblige l'Église à "sortir"77 à chercher des liens de fraternité et de solidarité universels et à savoir rester enracinée dans son milieu propre. Et n'oublions pas que nous avons aussi souligné que la charité vécue était un moyen sûr d'incarner la Bonne Nouvelle. Le pluralisme religieux est un défi et une chance. Mais c'est aussi un environnement exigeant, un regard extérieur qui oblige à être vraiment soi-même. C'est dans la mesure où la communauté chrétienne libanaise saura aller au plus profond du mystère de l'être humain et au plus près du mystère de Dieu, qu'elle pourra mener à bien une évangélisation digne de l'être humain et digne de la mission qui lui a été donné de vivre dans cette partie du monde. L'effort d'invention et d'adaptation aux attentes d'aujourd'hui n'est pas à négliger et peut permettre que l'expérience de foi des uns éclaire le chemin des autres et que, ensemble, l'Église d'Orient et l'Église d'Occident, discernent les signes de l'Esprit. "Allez donc" (Mt 28,19), car après tout c'est le Christ qui nous précède.

\section{Références bibliographiques}

BOSCH, D. J. Dynamique de la mission chrétienne. Histoire et avenir des modèles missionnaires. Paris-Lomé-Genève: Karthala-Haho-Labor et Fides, 1995.

CLAIR, A. Pseudonymie et paradoxe. La pensée dialectique de Kierkegaard. Paris: Librairie philosophique J. Vrin, 1976.

CONCILE VATICAN II. Constitutions - décrets - déclarations. Paris: éditions du Cerf, 1967.

CONGREGATION POUR L'EVANGELISATION DES PEUPLES. L'Eglise du Christ en Mission dans le Monde. Milano: San Paolo, 2019. (Oeuvres

${ }^{77}$ EG 20. 
Pontificale missionnaires) Disponible en: <http://www.october2019. $\mathrm{va/content/dam/october2019/documenti/la-guida-mmsott2019/Interno}$ Mese\%20Missionario\%20-\%20FRA\%20-\%20WEB.pdf>. Accès en: 23 jui. 2020.

CONSEIL CECUMÉNIQUE DES EGLISES. La mission et l'Evangélisation, affirmation oecuménique. Geneve: [s.n.], 1990.

COULON, P., La Mission Chrétienne De Vatican II À Aujourd'hui. Jalons Historiques Du Côté Catholique. Histoire et Missions Chrétiennes, v. 1, n. 1, p. 105-118, 2007.

DÉCLARATION de Lausanne. Perspectives Missionnaires, Lausanne, n. 2 , p. 66-75, 1981.

DELORME, J. La Résurrection de Jésus dans le langage du Nouveau Testament. Paris: Cerf, 1972 (LD, 72).

FRANÇOIS, PP. Exhortation Apostolique Evangelii Gaudium. Vatican, 2013. Disponible en: <http://www.vatican.va/content/francesco/fr/apost exhortations/documents/papa-francesco_esortazione-ap_20131124_ evangelii-gaudium.html >. Accès en: 23 jui. 2020.

HENDRIKUS, B. Christian Faith. Grand Rapids: Eerdmans, 1979.

JEAN-PAUL II, PP. Lettre encyclique Centesimus Annus. Vatican, 1991. Disponible en: <http://www.vatican.va/content/john-paul-ii/fr/encyclicals/ documents/hf_jp-ii_enc_01051991_centesimus-annus.html>. Accès en: 23 jui. 2020.

JEAN-PAUL II, PP. Lettre encyclique Redemptor Hominis. 1979. Disponible en: $<$ http://www.vatican.va/content/john-paul-ii/fr/encyclicals/documents/hf_ jp-ii_enc_04031979_redemptor-hominis.html>. Accès en: 23 jui. 2020.

JEAN-PAUL II, PP. Lettre encyclique Redemptoris Missio. 1991. Disponible en: $<$ http://www.vatican.va/content/john-paul-ii/fr/encyclicals/documents/hf_ jp-ii_enc_07121990_redemptoris-missio.html>. Accès en: 23 jui. 2020.

L'ÉVANGÉLISATION selon le pape François, Église Catholique en Alsace, 05 dec. 2017. Disponible en: $<$ https://www.alsace.catholique.fr/actualites/335697levangelisation-selon-le-pape-francois/>. Accès en: 23 jui. 2020.

LEGRAND, L. Le Dieu qui vient. La mission dans la Bible. Paris: Desclée, 1988. 
MARTIN-ACHARD, R. Israël et les nations. La perspective missionnaire de l'Ancien Testament. Neuchâtel-Paris: Delachaux et Niestlé, 1959 (Cahiers théologiques, 42).

MOULIN, L. Croyances et non-croyance. Cité du Vatican: publié par le secrétariat pour les non-croyants, 1984. v.XIX/2.

PATRIARCHES CATHOLIQUES D'ORIENT. La présence chrétienne en Orient, Mission et témoignage adressé à leurs fidèles, en Orient et dans la diaspora. Bkerke: [s.n.], 1992.

PAUL VI, PP. Exhortation apostolique Evangelii nuntiandi. Téqui: Éditions Pierre, 1975.

PAUL VI, PP. Lettre encyclique Ecclesiam suam. Vatican, 1964. Disponible en: <http://www.vatican.va/content/paul-vi/fr/encyclicals/documents/hf_pvi_enc_06081964_ecclesiam.html>.Accès en: 23 jui. 2020.

PIVOT, M. Un nouveau souffle pour la mission. Paris: Les éditions ouvrières, 2000.

RIGAL, J. Découvrir l'Église. Paris: Descellée de Brouwer, 2000.

SECRÉTARIAT POUR LE NON-CHRÉTIENS. Dialogue et la mission. 1984. Bulletin du Secrétariat pour le non-chrétiens. Attitude de l'Église devant les croyants des autres religions. Réflexions et orientations concernant le Dialogue et la Mission. Secrétariat pour le non-chrétiens, n. 56, p. 146-151, 1984.

SIMON, H. "L'Église naît de l'avenir...". La Croix, Samedi 2-Dimanche 3 et Lundi 4 juin 2001, p. 7.

SIMON, H. Vers une France Païenne? Paris: Cana, 1999.

SWIDLER, L. Understading Dialogue. Journal of Ecumenical Studies, v. 43, n. 2, p. 9-24, 2008.

SWIDLER, L. Deep-Dialogue/Critical-Thinking/Competitive-Cooperation: The Most Authentic Human Way To Be And Act. Journal of Ecumenical Studies, v. 47, n. 2, p. 143-151, 2012.

TESSIER, H. M. Vivre la mission de l'Église dans la maison de l'Islam. Spiritus, n. 94, p. 33-41, 1984.

TRILLING, W. Pauvreté. In: FRIES, H. (Dir.). Encyclopédie de la foi. Paris: Cerf, 1967. p. 371-375. t. XX. 
VERGOTE, H.-B. Dialectique de la communication, introduction et traduction de deux leçons de S. Kierkegaard comme pivat-docent. Revue de métaphysique et de morale, v. 76, n. 1, p. 53-76, jan./mar. 1971.

VIDAL, M. La nouvelle évangélisation. Spiritus, n. 145, p. 37-47, 1990.

WIDMER, G. Quelques repères d'une théologie de l'évangélisation. Revue de Théologie et de philosophie, v. 114, n. 4, p. 383-394, out./dez. 1982.

Ziad Fahed

Directeur du Bureau de la Mission Professeur Associé - Département d'études religieuses, culturelles et philosophiques Faculté des Sciences Humaines Notre Dame University de Louaize Liban E-mail: ziadfahed@ndu.edu.lb

Recebido em: 23/07/2020 Aprovado em: 24/02/2021 